\title{
Influence of the Overlap Coefficient on the Contrast in Laser Marking of C110W Steel
}

\author{
Nikolay Angelov \\ Technical University of Gabrovo \\ Gabrovo, Bulgaria \\ angelov_np@abv.bg
}

\author{
Lyubomir Lazov \\ Rezekne Academy of Technology \\ Rezekne, Latvia \\ lyubomir.lazov@rta.lv
}

\author{
Edmunds Teirumnieks \\ Rezekne Academy of Technology, \\ Rezekne, Latvia \\ edmunds.teirumnieks@rta.lv
}

\begin{abstract}
The laser marking process by melting samples of C110W carbon tool steel was studied. The experiments were performed with a fiber laser and a $\mathrm{CuBr}$ laser. A field of squares is marked in a raster method for different values of the overlap coefficient and power density. The contrast of the marking is determined on each marked square. From the obtained experimental data, graphs of the dependence of the contrast on the overlap coefficient for three power densities were drawn. The obtained results for the two lasers are compared and the influence of the wavelength is indirectly analysed. The working intervals of the overlap coefficient for the studied power densities for the two lasers at which the optimal contrast in the processing zone is obtained are determined.
\end{abstract}

Keywords - laser marking, fiber laser, CuBr laser, C110W steel, overlap coefficient, power density, contrast.

\section{INTRODUCTION}

Laser marking is a modern technology that is increasingly used in modern production in electronics, electrical engineering, mechanical engineering, medicine, and others. One of its main advantages is that it allows manufacturers to meet modern requirements for tracking and quality control of their products. Laser marking of serial numbers, matrix codes, barcodes, logos, tables, and other operational information is the main factor for proper optimization and monitoring of production processes $[1 \div$ 3], [11 $\div$ 17]. The wide penetration of laser marking technology in industrial production is primarily due to its special characteristics, such as non-contact during the process, which allows processing both ultra-hard materials for mechanical engineering and brittle and thin materials for microelectronics. The selectivity of the laser interaction with the material combined with the simultaneous ability to achieve heating, melting, and evaporation of the material in a very small area is another significant advantage of this innovative technology over other marking methods. The ability of modern laser scanners to control the movement of the laser beam in space at high speed and to position it with high accuracy is another important factor that has significantly contributed to the entry of this technology in the modern production of various types of products [4].

Laser marking is a complex technological process that depends on a number of factors that can be combined into three main groups $[5,6]$. It can be noted that these factors in most cases are interrelated and have a complex effect on the specific technological process [7]. The article [8] with a nanosecond pulsed laser analyses the influence of the pulse tracking frequency and the distance between the processing lines when scanning titanium alloy substrates on the process of colour laser marking. The authors in [9] investigated the effect of a number of parameters of laser processing, such as power and fill factor of laser pulses on a certain working area when marking barcodes on the surfaces of aluminum alloys. The results show that the processing parameters significantly affect the surface roughness.

The study [10] demonstrated a processing technique related to the overlap of the laser pulses and the overlap between the scanning lines influencing the formation of specific microstructures on the surface of Ti6Al4V samples. The topology of processing in the process considered by the authors varies in a wide range between $40 \%$ and $90 \%$ and leads to a change in the density of the absorbed laser energy in the range from $0.49 \mathrm{~J} / \mathrm{cm}^{2}$ to

\section{Online ISSN 2256-070X \\ https://doi.org/10.17770/etr2021vol3.6599 (C) 2021 Nikolay Angelov, Lybomir Lazov, Edmunds Teirumnieks. Published by Rezekne Academy of Technologies.} This is an open access article under the Creative Commons Attribution 4.0 International License. 
$12.28 \mathrm{~J} / \mathrm{cm}^{2}$ and this in turn to optimization of the quality of the technological process.

The present study focuses on the analysis and the relationship between two technological factors, the overlap coefficient and the scan overlap coefficient, on the quality of the marking and in particular on the contrast in the processing area. Their influence has a direct impact on the linear density of the pulses entering at the processing area, as well as on the linear energy density and the effective energy for the realization of the specific technological process.

\section{TOPOLOGY OF THE EXPERIMENTAL STUDY}

The overlap coefficients $k_{o v}$ of the laser pulses and the overlap coefficient $k_{s}$ in the scanning process are complex factors that give us a relationship between the marking speed (factor related to the technological process), on the one hand, and the pulse repetition rate and laser power density in diameter. at the workplace (factors related to the parameters of the laser) on the other hand.

Considering that, the physical interaction of the laser radiation with the substance in the treatment area leading to heating, melting and evaporation is strongly influenced by the absorbed energy (effective energy for the specific technological process $E_{\text {eff }}$ ).

Considering that the linear energy density at laser action is the quantity defined by the absorbed energy per unit length in the laser marking area

$$
E_{\ell}=\frac{A P}{v}
$$

where $v$ is the marking speed, $P$ is power and $A$ is absorption capacity.

In the case of pulsed raster marking, as in our study, the overlap coefficient $k_{o v}$ significantly affects the value of the linear pulse density of laser pulses entering the processing line

$$
L_{p}=\frac{v}{v},
$$

where $v$ is the frequency of pulse repetition.

In turn, the above two relations (1) and (2) have a significant influence on the effective absorbed energy of the laser radiation per unit area of the laser impact zone

$$
E_{\text {eff }}=\frac{A P v}{v^{2}}
$$

and on the volume absorbed energy of the laser radiation

$$
E_{\rho}=\frac{A P}{d v \delta}
$$

where $\delta$ is the penetration of laser radiation into the substance.

Taking into account all these interrelated relations between the physical quantities and the technological parameters of the processing of formulas (1) - (4) it is seen that the topology of the experiment is essential for the expected result of the processing.

The overlap coefficient $k_{o v}$ of the laser radiation pulses is a complex factor that gives a relationship between the marking speed (factor related to the technological process) on the one hand and the frequency of pulse repetition and laser power density through the diameter of the working spot (factors related to the laser parameters) on the other hand. It is defined by the expression

$$
k_{o v}=\frac{x}{d} \cdot 100 \%=\left(1-\frac{\ell}{d}\right) \cdot 100 \%,
$$

where $d$ is the diameter of the working spot, $x$ is the width of the overlapped part of two consecutive pulses of laser radiation, $\ell$ is the distance between two consecutive pulses (Fig. 1).

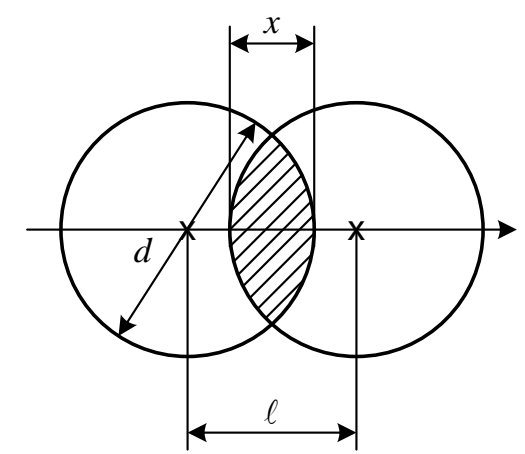

Fig. 1. Position of two consecutive pulses in the presence of overlap

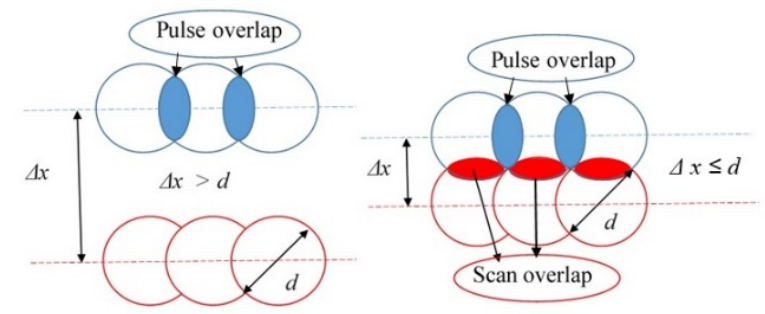

Fig. 2. Intervals of change of the scan overlap coefficient $k_{s}$

Taking into account that

$$
\ell=\frac{v}{v}
$$

where $v$ is the marking speed and $v$ is the frequency of pulse repetition, and replacing (2) in (1) is obtained

$$
k_{\text {ov }}=\left(1-\frac{v}{v d}\right) \cdot 100 \%
$$

The laser marking process also is affected on the scan overlap coefficient $k_{s}$. It depends on the diameter $\mathrm{d}$ of the working spot and the raster step $\Delta x$. The scan overlap coefficient is given by the formula 


$$
k_{s}=\left(1-\frac{\Delta x}{d}\right) \cdot 100 \%
$$

When the condition $\Delta x \leq d$ is fulfilled, it has positive values and is in the interval $k_{s} \in[0,100] \%$. When the condition $\Delta x>d$ is fulfilled, it takes negative values. The limitation for raster marking is that the consecutive lines are at a smaller distance from the limiting ability of the human eye, i.e. $\Delta x \leq 70 \mu \mathrm{m}$. In this case, the homogeneity of the marking is not disturbed.

It should be noted that there is a relationship between some of the considered physical quantities and technological parameters. For example, the overlap coefficient $k_{o v}$ can be related to the linear pulse density $L_{p}$ and the effective energy $E_{\text {eff }}$ with the expressions

$$
\begin{array}{r}
k_{o v}=\left(1-\frac{1}{L_{p} d}\right) \cdot 100 \% \\
\text { and } k_{\text {ov }}=\left(1-\frac{A P}{E_{\text {eff }} v d}\right) \cdot 100 \%
\end{array}
$$

\section{EXPERIMENTS AND DISKUSIONS}

The experiments refer to C110W carbon tool steel. It is widely used in industry. C110W steel is used for making hand taps, rasps, needle files, saws for woodworking, matrices for cold stamping. It is high carbon steel and has relatively high coefficients of thermal conductivity and diffusivity (see Table 1).

TABLE 1. BASIC CHARACTERISTICS OF THE C110W CARBON TOOL STEEL

\begin{tabular}{|l|l|}
\hline Characteristic & Value \\
\hline Coefficient of thermal conductivity $k, \mathrm{~W} /(\mathrm{m} . \mathrm{K})$ & 45 \\
\hline Specific heat capacity $c, \mathrm{~J} /(\mathrm{kg} . \mathrm{K})$ & 460 \\
\hline Density $\rho, \mathrm{kg} / \mathrm{m}^{3}$ & 7850 \\
\hline Coefficient of diffusivity $a, \mathrm{~m}^{2} / \mathrm{s}$ & $1.25 \times 10^{-5}$ \\
\hline
\end{tabular}

The experimental studies were performed with laser technological systems for marking with fiber laser and $\mathrm{CuBr}$ laser. Their main parameters are presented in Table 2.

TABLE 2 SOME BASIC PARAMETERS OF A LASER TECHNOLOGICAL SYSTEM FOR MARKING WITH FIBER LASER TYPE STYLES

\begin{tabular}{|l|l|l|}
\hline Parameter & Fiber laser & CuBr laser \\
\hline Wavelength $\lambda, \mathrm{nm}$ & 1064 & $511 \& 578$ \\
\hline Power $P, \mathrm{~W}$ & 20.0 & 10.0 \\
\hline Frequency $v, \mathrm{kHz}$ & 80 & 20 \\
\hline Pulse duration $\tau, \mathrm{ns}$ & 100 & 30 \\
\hline Pulse energy $E_{p}, \mathrm{~mJ}$ & 0.25 & 0.50 \\
\hline Pulse power $P_{p}, \mathrm{~kW}$ & 2.50 & 16.7 \\
\hline Quality of beam $\mathrm{M} 2$ & $<1.1$ & $<1.7$ \\
\hline Positioning accuracy, $\mu \mathrm{m}$ & 2.5 & 2.5 \\
\hline Efficiency, $\%$ & 40 & 10 \\
\hline
\end{tabular}

The fiber laser is a modern laser emitting in the near infrared range. It works in pulse mode. It has extremely high beam quality, high efficiency. and cheap maintenance. The laser system has high precision and very good repeatability.

The $\mathrm{CuBr}$ laser emits in the visible range. It works in pulse mode. It is characterized by good beam quality and relatively good efficiency. The laser system has high precision and maintains stable parameters during operation.

During the experiments, the scan overlap coefficient $k_{s}$ remained constant. Squares with a side of $5 \mathrm{~mm}$ were marked in a raster method way. The contrast was determined several times for each marked square. The contrast was measured according to the procedure described in [7]. The mean contrast value for each square was then obtained.

The experiments were conducted in two directions:

1. Investigation of the dependence of the contrast of the marking $k^{*}$ on the overlap coefficient $k_{o v}$ for fiber laser

A series of experiments was carried out in order to study the influence of the overlap coefficient kov on the contrast $k^{*}$ of the marking. The overlap coefficient changes in the interval $k_{o v} \in[72.5 \div 95]$ \% through $2.50 \%$. Experiments refer for three values of the power density: $q_{s 1}=1.24 \times 10^{10}$ $\mathrm{W} / \mathrm{m}^{2} ; q_{\mathrm{s} 2}=1.50 \times 10^{10} \mathrm{~W} / \mathrm{m}^{2}$ and $q_{\mathrm{s} 3}=1.77 \times 10^{10} \mathrm{~W} / \mathrm{m}^{2}$. The parameters that are kept constant are given in Table 3 . During the experiments, the scan overlap coefficient had value $k_{s}=-25 \%$.

TABLE 3. PARAMETERS THAT DO NOT CHANGE DURING EXPERIMENTS

\begin{tabular}{|l|l|l|}
\hline \multicolumn{1}{|c|}{ Laser } & Fiber & $\mathbf{C u B r}$ \\
\hline Parameter & & \\
\hline Diameter of working spot $d, \mu \mathrm{m}$ & 100 & 40 \\
\hline Raster step $\Delta x, \mu \mathrm{m}$ & 40 & 30 \\
\hline Number of repetition & 50 & 50 \\
\hline Defocusing $\Delta f, \mathrm{~mm}$ & 1 & 1 \\
\hline
\end{tabular}

The obtained experimental dependencies of the contrast $k^{*}$ of the marking from the overlap coefficient kov are presented in graphical form in Fig. 3.

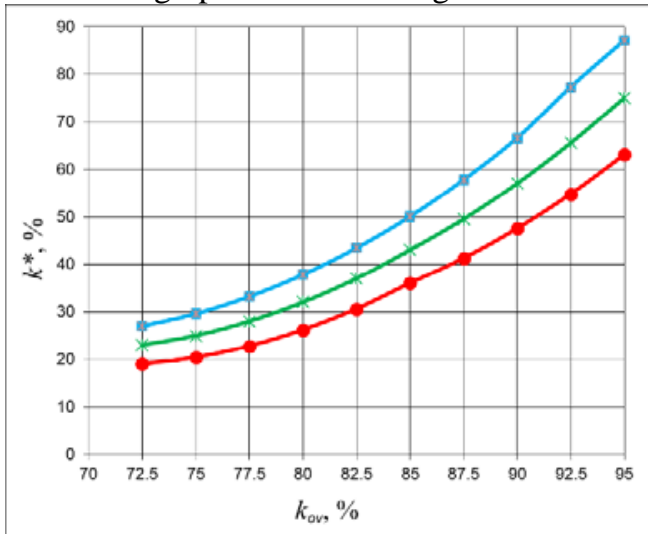

Figure 3. Dependence of the contrast on the overlap coefficient for marking with a fiber laser for power densities: red colour $-q_{\mathrm{s} 1}=$ $1.24 \times 10^{10} \mathrm{~W} / \mathrm{m}^{2}$; green colour $-q_{\mathrm{s} 2}=1.50 \times 10^{10} \mathrm{~W} / \mathrm{m}^{2}$; blue colour $-q_{\mathrm{s} 3}$ $=1.77 \times 10^{10} \mathrm{~W} / \mathrm{m}^{2}$. 
From the analysis of experimental results it sees that:

- With increasing the overlap coefficient results in a non-linear increase in contrast for all three power density. In the interval $k_{o v} \in[80 \div 95] \%$ the contrast $k^{*}$ increases significantly faster versus the interval $k_{o v}$ $\mathrm{C}[72.5 \div 80] \%$;

- The rate of increase of the contrast $k^{*}$ is given in the table below.

\begin{tabular}{|c|c|c|l|l|l|}
\hline \multicolumn{4}{|c|}{ The rate of increase of the contrast $\boldsymbol{k}^{*}$} \\
\hline \multicolumn{3}{|c|}{ interval $k_{o v}[\mathbf{7 2 . 5} \div \mathbf{8 0}] \%$} & \multicolumn{3}{|c|}{ interval $k_{o v}[\mathbf{8 0} \div \mathbf{9 5}] \%$} \\
\hline $\mathbf{q}_{\mathbf{s} 1}$ & $\mathbf{q}_{\mathbf{s} 2}$ & $\mathbf{q}_{\mathbf{s} 3}$ & $\mathbf{q}_{\mathbf{s} 1}$ & $\mathbf{q}_{\mathbf{s} 2}$ & $\mathbf{q}_{\mathbf{s} 3}$ \\
\hline $\mathbf{0 . 9 6}$ & $\mathbf{1 . 2 0}$ & $\mathbf{1 . 4 4}$ & $\mathbf{2 . 4 5}$ & $\mathbf{2 . 8 7}$ & $\mathbf{3 . 2 8}$ \\
\hline
\end{tabular}

The faster increase of the contrast in the interval $k_{o v} \mathrm{C}$ [80 $\div 95] \%$ compared to the interval $k_{o v} \in[72.5 \div 80]$ \%kov is explained by the fact that with increasing the overlap coefficient the absorbency of the material in the impact zone also increases.

- The following working intervals were determined for the overlap coefficient $k_{\mathrm{ov}}$.

\begin{tabular}{|l|l|}
\hline for power density $\boldsymbol{q}_{\mathbf{s}}, \mathbf{W} / \mathbf{m}^{2}$ & the overlap coefficient $\boldsymbol{k}_{\boldsymbol{o v}}$ \\
\hline $1.24 \times 10^{10}$ & $91 \% \div 95 \%$ \\
\hline $1.50 \times 10^{10}$ & $87.5 \% \div 95 \%$ \\
\hline $1.77 \times 10^{10}$ & $85 \% \div 95 \%$ \\
\hline
\end{tabular}

These intervals refer to the visual perception of the marking, for which the contrast must be at least $50 \%$.

\section{Investigation of the dependence of the contrast $\boldsymbol{k}^{*}$ of the marking on the overlap coefficient $\mathrm{k}_{o v}$ for $\mathrm{CuBr}$ laser}

A number of experiments was carried out in order to study the influence of the overlap coefficient $k_{o v}$ on the contrast $k^{*}$ of the marking. The overlap coefficient changes in the interval $k_{o v} \in[63.33 \div 93.33] \%$ with step $3.33 \%$. The experiments were performed at three constant values of power density $q_{s 1}=0.99 \times 10^{10} \mathrm{~W} / \mathrm{m}^{2} ; q_{\mathrm{s} 2}=$ $1.20 \times 10^{10} \mathrm{~W} / \mathrm{m}^{2}$ and $q_{\mathrm{s} 3}=1.42 \times 10^{10} \mathrm{~W} / \mathrm{m}^{2}$. The parameters that are kept constant are given in Table 3 . During the experiments, the scan overlap coefficient had value $k_{\mathrm{s}}=-66.7 \%$

The obtained experimental dependencies of the contrast $k^{*}$ of the marking from the overlap coefficient $k_{o v}$ are presented in graphical form in Fig. 4. From the analysis of experimental results, it sees that:

- With increasing the overlap coefficient results in a non-linear increase in contrast for all three power density. In the interval $k_{o v} \in[73.3 \div 93.3] \%$ the contrast $k^{*}$ increases significantly faster versus the interval $k_{o v} \in[63.3 \div 73.3] \%$;

- When working with a $\mathrm{CuBr}$ laser, the optimal contrast of the marking is achieved with lower values of power density $q_{s}$ and overlap coefficient than when marking with a fiber laser. It is explained that $\mathrm{CuBr}$ laser radiation $(\lambda=511 \mathrm{~nm} \& 578 \mathrm{~nm})$ is better absorbed by the studied material than fiber laser radiation $(\lambda=$ $1064 \mathrm{~nm})$.

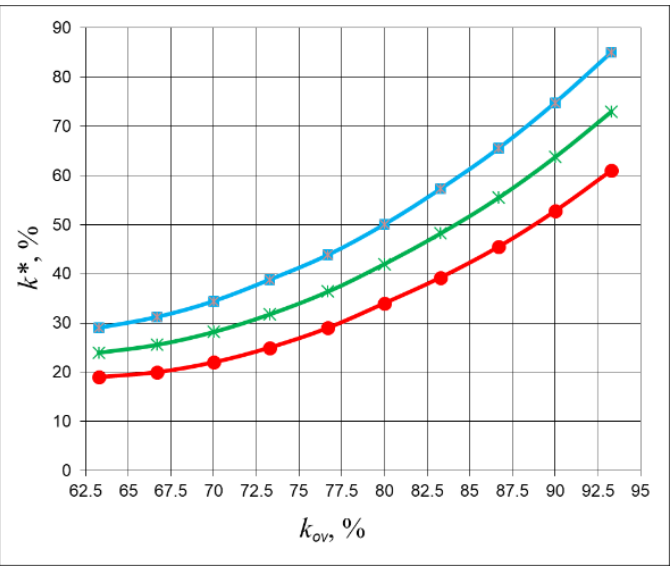

Figure 4. Dependence of the contrast on the overlap coefficient for marking with a $\mathrm{CuBr}$ laser for power densities: red colour $-q_{\mathrm{s} 1}=$ $0.99 \times 10^{10} \mathrm{~W} / \mathrm{m}^{2}$; green colour $-q_{\mathrm{s} 2}=1.20 \times 10^{10} \mathrm{~W} / \mathrm{m}^{2}$; blue colour $-q_{\mathrm{s} 3}$ $=1.42 \times 10^{10} \mathrm{~W} / \mathrm{m}^{2}$.

- The rate of increase of the contrast $k^{*}$ is given in the table below.

\begin{tabular}{|c|c|c|l|l|l|}
\hline \multicolumn{5}{|c|}{ The rate of increase of the contrast $\boldsymbol{k}^{*}$} \\
\hline \multicolumn{3}{|c|}{ interval $k_{o v}[\mathbf{6 3 . 3} \div \mathbf{7 3 . 3} \% \%$} & \multicolumn{3}{|c|}{ interval $k_{o v}[\mathbf{7 3 . 3} \div \mathbf{9 3 . 3}] \%$} \\
\hline $\boldsymbol{q}_{\mathrm{s} 1}$ & $\boldsymbol{q}_{\mathrm{s} 2}$ & $\boldsymbol{q}_{\mathrm{s} 3}$ & $\boldsymbol{q}_{\mathrm{s} 1}$ & $\boldsymbol{q}_{\mathrm{s} 2}$ & $\boldsymbol{q}_{\mathrm{s} 3}$ \\
\hline $\mathbf{0 . 6 0}$ & $\mathbf{0 . 7 8}$ & $\mathbf{0 . 9 8}$ & $\mathbf{1 . 8 0}$ & $\mathbf{2 . 0 6}$ & $\mathbf{2 . 3 1}$ \\
\hline
\end{tabular}

Here again, the different rate of contrast increase is due to the dependence of the absorbency of the material on the overlap coefficient.

- The following working intervals were determined for the overlap coefficient $k_{o v}$ for visual perception of the marking.

\begin{tabular}{|l|l|}
\hline for power density $\mathbf{q}_{\mathbf{s}}, \mathbf{W} / \mathbf{m}^{2}$ & the overlap coefficient $\boldsymbol{k}_{\boldsymbol{o v}}$ \\
\hline $0.99 \times 10^{10}$ & $88.5 \% \div 93.3 \%$ \\
\hline $1.20 \times 10^{10}$ & $84 \% \div 93.3 \%$ \\
\hline $1.42 \times 10^{10}$ & $80 \% \div 93.3 \%$ \\
\hline
\end{tabular}

\section{CONCLUSIONS}

This study examines the effect of laser parameters on contrast in the area of marking. The processing field (squares) is marked using the raster method at different values of the overlap coefficient between the individual laser pulses and the overlap coefficient between the scanning lines. The research is related to optimizing the influence of the overlap coefficient on the marking process and more precisely on the contrast. The results and analyses of this study have a direct impact on the optimization and 
quality of application of two-dimensional QR codes on C110W carbon tool steel surfaces with two types of lasers fiber and $\mathrm{CuBr}$.

\section{REFERENCES}

[1] Grigoryanc, A., Shiganov, I. \& Misyurov, A., Technological processes of laser pro-cessing, Publ. MGTU N. Baumana, Moskow, 2006

[2] Lazov L. K., N. A. Petrov, Investigation of the Impact of the Number of Repetitions and Defocus on the Contrast of Laser Marking for Products Made of Tool Steel, Metalofizika I noveishie Tekhnologii, t.34, № 7, pp. 1003-1011, 2012, ISBN 1024-1809

[3] Sobotova, L., P. Demec, Laser Marking of Metal Materials, Modern Machinery Science Jounal, 808-812, 2015.

[4] E. Nikolidakis, I. Choreftakis, A. Antoniadis, Experimental Investigation of Stainless Steel SAE304 Laser Engraving Cutting Conditions, Machines, 6, 40; 1-8, 2018, DOI:10.3390/machines6030040

[5] Lazov L., N. Angelov, Basic factors determining quality of markings on metals and alloys. International Scientific Conference AMTECH - 2007, Gabrovo, Vol. I, 102-107, 2007

[6] Lazov L., A. Atanasov, N. Angelov, Investigation of the role of certain factors affecting the contrast in laser marking, Contemporary Materials, Vol. II-1, 13-17, 2011.

[7] Angelov, N., Optimization of the process of laser marking of samples of tool steel, dissertation for the degree of DOCTOR, TU - Gabrovo, 2011

[8] L. Chunling, L. Changhou, Laser-Induced Color Marking of Titanium Alloy, TELKOMNIKA, Vol.14, No.4, 1313-1320, 2016, ISSN: 1693-6930, DOI: 10.12928/TELKOMNIKA.v14i4.4177

[9] L. Jianmei, L. Changhou, W. Aiqun, W. Yusong, M. Zhuo, F. Xinxin, T. Liang, Experimental investigation and mathematical modeling of laser marking two-dimensional barcodes on surfaces of aluminum alloy, Journal of Manufacturing Processes 21, 141152, 2016, DOI: https://doi.org/10.1016/j.jmapro.2015.12.007
[10] G. Schnell, U. Duenow, H. Seitz, Effect of Laser Pulse Overlap and Scanning Line Overlap on Femtosecond Laser-Structured Ti6Al4V Surfaces, Materials 2020, 13, 969; 1-15, DOI:10.3390/ma13040969

[11] Dolchinkov, N., Practical Research of Marking and Cutting of Textiles with Increased Resistance, Using CO2 Laser, Journal of Physics: Conference Series, Volume 1681, 2020012014 IOP Publishing DOI:10.1088/1742-6596/1681/1/012014

[12] Kolev, I., T. Karadzhov, Classification, parameters, characteristics and application of matrix semiconductor photoconverters, 27th International Spring Seminar on Electronics Technology, vol. 3, 444-447, 2004, DOI: 10.1109/ISSE.2004.1490852

[13] Pencheva T., D. Pulov, B. Gyoch, M. Nenkov, Design of CCD Optical System for Thermal IR Spectral Region. In pr. 29-th International Spring Seminar fn Electronics Technology, St. Marienthal, Germany, Verlag Dr. Markus A. Detert, 413-418, 2006, ISBN 3-934142-23-0, DOI: 10.1109/ISSE.2006.365380

[14] Dolchinkov, N., Investigation of the State of the Radiation Control Systems and the Actions of the Competent Authorities and the Population in the Event of a Change in the Radiation Background in Bulgaria, International Conference KNOWLEDGE-BASED ORGANIZATION, Vol. XXIV No 3, 38-44, 2018, DOI: https://doi.org/10.1515/kbo-2018-0134

[15] Lazov L, N. Angelov, Physical model about laser impact on metals and alloys, Contemporary Materials, Vol. I-2, 124-128, 2010.

[16] Valiulin A., S. Gorniy,Y. Grechko, M. Patrov, K. Yudin, V. Yurevich, Laser marking of metals, Scientific and technical journal "Photonics",, №3, 16-22, 2007.

[17] Cvetanov, G., T. Karadzhov, R. Miteva, Determination of the elastic displacements in plain strained condition of involute cylindrical gears with asymmetric profile. AIP Conference Proceedings, Volume 2333, 2021, DOI: 10.1063/5.0042511 